\title{
Homogenization of contact line dynamics
}

\author{
K. B. GLASNER ${ }^{\dagger}$ \\ Department of Mathematics, The University of Arizona, \\ 617 N Santa Rita, Tucson, AZ 85721, USA
}

[Received 20 February 2006 and in revised form 12 May 2006]

\begin{abstract}
This paper considers the effects of substrate inhomogeneity on the motion of the three phase contact line. The model employed assumes the slowness of the contact line in comparison to capillary relaxation. The homogenization of this free boundary problem with a spatially periodic velocity law is considered. Formal multiple scales analysis yields a local, periodic problem whose time-averaged dynamics corresponds to the homogenized front velocity. A rigorous understanding of the long time dynamics is developed using comparison techniques. Computations employing boundary integral equations are used to illustrate the consequences of the analysis. Advancing and receding contact angles, pinning and anisotropic motion can be predicted within this framework.
\end{abstract}

In many realistic circumstances, the static and dynamic wetting properties of liquids are substantially influenced by imperfections in the solid surface. Heterogeneities result in contact lines with a fine scale structure that may lead to pinning of the evolving front and hysteresis of the overall fluid shape.

Understanding the role that surface imperfections play is part of larger theoretical effort to determine the macroscopic manifestations of microscopic contact line features [4, 15, 42]. Classical fluid mechanics is by itself insufficient to describe the moving contact line [32], and the additional physical ingredients needed are still controversial. Modeling and theoretical studies include notions of slip boundary conditions [28, 30], continuum models [15, 45, 51], rheological modifications [27, [53], and atomistic simulations [19, 29] (see [4, 42] for more extensive accounts). There is also considerable technological importance in understanding the role of wetting on patterned substrates [11, 17, 37, 48].

The model studied here is based on the slowness of the contact line in comparison to the time for capillary relaxation, known as the quasistatic limit. This represents possibly the simplest nontrivial global model for contact line motion, and therefore provides a good point of departure for examining surface heterogeneity. In this limiting case, the fluid pressure is constant, and the fluid's geometry can therefore be described as a "capillary" surface. Motion arises from an imbalance of surface forces at the contact line itself, which can be modeled by a constitutive velocity-contact angle law. This approximation has been utilized in many previous studies [2, 21, 23, 28, 31, 33, 40, 43].

The static effects of surface heterogeneity have been studied for some time. Early heuristic theories considered the averaged effect of rough surfaces and chemical heterogeneities on the equilibrium contact angle [7, 54]. These laws have known limits of applicability, however ([4, Chapter 9], [3]). More recent studies have considered this problem from the point of view of mechanics [4, 34], statistics [14, 41], and gamma convergence techniques [1].

In the dynamic case, much less is understood. Flows over heterogeneous surfaces have been studied experimentally [11, 12, 38, 39, 47] and computationally [49, 50]. Theoretical arguments, on

${ }^{\dagger}$ E-mail: kglasner@math.arizona.edu 
the other hand, have been limited to cases with simplified geometries [35, 46] or deal specifically with the dynamic coating transition [26].

Homogenization of free boundary and front propagation problems is a young and evolving field (see, e.g., [5, 9, 55]). The strategy we adopt is a combination of formal asymptotic calculations coupled with a detailed understanding of the "inner" or "cell" problem. The existence and uniqueness of the homogenized velocity is studied rigorously. A Green's function formulation is used for numerical computation to illustrate the analytical results.

\section{Problem formulation}

We consider a fluid droplet on a macroscopically flat surface whose height is assumed to be a graph $h(x, y)$. The function $h$ has bounded support $\Omega$, whose boundary is the contact line $\Gamma$. In the small angle limit [6], the linearized contribution of surface energy is given by

$$
\gamma \int_{\Omega}\left(1+|\nabla h|^{2}\right) \mathrm{d} \boldsymbol{x}
$$

where $\gamma$ is the liquid-vapor surface energy, which is presumed constant here. The minimizer of this expression, subject to the constraint of constant volume, is the quasi-steady droplet shape. Finding the minimizer amounts to solving the Euler-Lagrange problem

$$
\Delta h=\lambda,\left.\quad h\right|_{\Gamma}=0, \quad \int_{\Omega} h \mathrm{~d} \boldsymbol{x}=M \equiv \text { volume },
$$

where $\lambda<0$ is a Lagrange multiplier, essentially just the negative hydrostatic pressure. We remark that body forces such as gravity can be added to this model [22] without compromising the homogenization procedure.

The "apparent" contact angle $\theta$ is defined on the boundary of $\Omega$ (again in the small angle limit) as

$$
\theta=-\frac{\partial h}{\partial \boldsymbol{n}}, \quad \boldsymbol{n}=\text { outward normal of } \Gamma .
$$

The motion of $\Gamma$ is then specified by prescribing the normal velocity, denoted $\mathrm{d} \Gamma / \mathrm{d} t$, as a function of this angle and the spatial location of the contact line:

$$
\frac{\mathrm{d} \Gamma}{\mathrm{d} t}=F(\theta ; \boldsymbol{x})
$$

This can be recast in terms of $h$ as the free boundary condition

$$
h_{t}=F(|\nabla h| ; \boldsymbol{x})|\nabla h|, \quad \text { when } h=0 .
$$

The function $F$ is a constitutive law relating the contact line velocity to the apparent contact angle $\theta$. Generally speaking, $F$ must be continuous and an increasing function of $\theta$ so that there is a local "equilibrium" angle $\theta_{e}(\boldsymbol{x})$ where $F\left(\theta_{e}(\boldsymbol{x}) ; \boldsymbol{x}\right)=0$. Some additional technical restrictions will be placed on $F$ to obtain the rigorous results of Section 4 Common examples found in the literature are

$$
\begin{array}{ll}
F=\theta^{3}-\theta_{e}^{3} & (\text { Cox [8] }, \text { Voinov [52] }) \\
F=\theta\left(\theta^{2}-\theta_{e}^{2}\right) & (\text { de Gennes [16] })
\end{array}
$$


The spatial dependence of $F$ arises as a consequence of substrate inhomogeneities. We focus here on two-dimensional periodicity with some small period $\epsilon$ :

$$
F(\theta ; \boldsymbol{x}+\epsilon \boldsymbol{k})=F(\theta ; \boldsymbol{x}) \quad \text { for all } \boldsymbol{k} \in \mathbb{Z}^{2} .
$$

The purpose of this paper is to examine the homogenized limit $\epsilon \rightarrow 0$ by deriving an effective velocity law that is independent of $\epsilon$.

The free boundary problem (2), (5) forms the basis for our study. While a complete and rigorous analysis has not been performed on this particular model, it has much in common with wellunderstood problems such as zero surface tension Hele-Shaw flow [13, 36]. This paper's scope is restricted just to smooth solutions of (2), (5). We anticipate that a generalized notion of nonsmooth solutions is possible, akin to the theory of viscosity solutions which has been developed for a variety of second order nonlinear problems [10, 18, 36].

\section{Formal multiple scales analysis}

The goal of this paper is to derive a free boundary problem of the form (2), (5) in the limit $\epsilon \rightarrow 0$, where the contact angle-velocity relation is replaced by a homogenized version

$$
\frac{\mathrm{d} \Gamma}{\mathrm{d} t}=F_{\mathrm{hom}}(\theta ; \boldsymbol{n})
$$

The dependence on the normal direction $\boldsymbol{n}$ to the contact line stems from the anisotropy introduced by periodicity of $F$. In particular, $F_{\text {hom }}$ will inherit the four-fold symmetry from the periodic heterogeneity. (Other types of periodicity, hexagonal, rhombic, etc. should of course lead to other types of symmetry in the homogenized velocity.) The strategy we adopt is to use a combination of spatial matched asymptotics and a multiple-scales ansatz in time to derive a dynamic "cell problem". This problem must be solved independently, either analytically or numerically, and is studied at length in later sections.

Homogenization of problems which have a fine-structured boundary have been considered previously. In the same spirit as this work, Gobbert \& Ringhofer [25] use a multiscale matched asymptotic expansion for the case where one of the boundaries has the form of a graph $y=$ $f(x)+f_{1}(x / \epsilon)$. They derive the limiting solution as solving the same elliptic problem with an effective boundary condition which they compute. Friedman \& Hu [20] considered a similar problem where there was a prescribed moving free boundary $y=f(x)+f_{1}(x / \epsilon, t)$ and proved convergence estimates for $\epsilon \rightarrow 0$. Our problem presents two additional challenges. The first is that the free boundary motion is coupled to the solution itself. One consequence of this is that the free boundary evolves with fine scale oscillations both in time and space, which motivates the use of a multiple scale expansion in time. The second complication is that the free boundary has a smooth but arbitrary geometry. To deal with this, we introduce a local coordinate system fitted to the asymptotic free boundary.

\subsection{Setup and motivation}

While the asymptotic analysis that follows is presented at a purely formal level, we provide some justification for the expansions as well as hint at what ingredients are necessary to make the calculation completely rigorous. The main assumptions which are needed to justify the asymptotic expansions are: 
1. Control over the free boundary. As the free boundary evolves, it necessarily develops a fine structure which we suppose has wavelength $\epsilon$ and (spatial) amplitude $\epsilon$. An argument in favor of this is given in the appendix, where a local bound on the front width is proved with respect to coordinates rescaled like $\epsilon^{-1}$. This implies that the spatial amplitude of free boundary oscillation should behave like $\mathcal{O}(\epsilon)$. We suppose that for each $t>0, \Omega(t ; \epsilon)$ remains simply connected and approaches a limiting set $\Omega_{0}(t)$ in the sense that its boundary converges at a suitable rate:

$$
\max _{\boldsymbol{x}_{1} \in \partial \Omega(t ; \epsilon)} \min _{\boldsymbol{x} \in \partial \Omega_{0}(t)} \operatorname{dist}\left(\boldsymbol{x}, \boldsymbol{x}_{1}\right)=\mathcal{O}(\epsilon)
$$

2. Regularity in the interior. Away from the free boundary, oscillations in $h$ should decay exponentially. As an illustration, suppose that the boundary is given by the graph of the function $y=\epsilon f(x / \epsilon)$ and consider the boundary value problem

$$
\begin{array}{rlrl}
\Delta u & =0, & y>\epsilon f(x / \epsilon), \\
u(x, f(x / \epsilon)) & =0, & \\
\frac{\partial u}{\partial y} & \rightarrow 1, \quad y \rightarrow \infty .
\end{array}
$$

If we introduce the rescaling $\left(x^{\prime}, y^{\prime}\right)=\epsilon^{-1}(x, y)$, then $u^{\prime}\left(x^{\prime}, y^{\prime}\right)=\epsilon^{-1} u\left(\epsilon x^{\prime}, \epsilon y^{\prime}\right)-y^{\prime}$ solves the $\epsilon$-independent problem

$$
\begin{aligned}
\Delta u^{\prime} & =0, \quad y^{\prime}>f\left(x^{\prime}\right), \\
u^{\prime}\left(x^{\prime}, f\left(x^{\prime}\right)\right) & =-f\left(x^{\prime}\right), \\
\frac{\partial u^{\prime}}{\partial y^{\prime}} & \rightarrow 0, \quad y^{\prime} \rightarrow \infty .
\end{aligned}
$$

One can show (e.g. [44]) that $u^{\prime}$ is periodic in $y^{\prime}$ and all its derivatives decay exponentially in $y^{\prime}$ as $y^{\prime} \rightarrow \infty$. In particular, for the unscaled problem there is a bound on second derivatives of the form

$$
\left|D^{2} u\right| \leqslant C_{1}+C_{2} \epsilon^{-1} \exp (-y / \epsilon) \quad \text { for } y>C_{3} \epsilon
$$

where $C_{n}$ are constants independent of $\epsilon$. We might therefore expect that our problem also enjoys a similar bound of the form

$$
\left|D^{2} h(\boldsymbol{x})\right| \leqslant C_{1}+C_{2} \epsilon^{-1} \exp (-d / \epsilon), \quad d=\operatorname{dist}(\Gamma, \boldsymbol{x}), \quad \text { for } d>C_{3} \epsilon .
$$

Of course, the precise justification for this relies on the detailed regularity properties for solutions of (2)-(5), which is not within this paper's scope.

3. Control over $\nabla h$ near the boundary. We need to ensure that, near the boundary, $|\nabla h|$ is bounded away from zero as we take $\epsilon \rightarrow 0$. This is basically equivalent to assuming that the free boundary remains smooth. Indeed, the Hopf maximum principle (which requires a smooth boundary) applied to $h-\lambda\left|\boldsymbol{x}-\boldsymbol{x}_{0}\right|^{2} / 4$ yields $(\mathrm{d} h / \mathrm{d} n)\left(\boldsymbol{x}_{0}\right)<0$. Conversely, if the homogenized solution is smooth enough and $\mathrm{d} h / \mathrm{d} n<0$ at all points on the boundary, then the zero level set of $h$ is given by a smooth parameterized curve $\Gamma=\boldsymbol{x}(s)$ via the differential equation

$$
\frac{\mathrm{d}}{\mathrm{d} s} h(\boldsymbol{x}(s))=\nabla h \cdot \frac{\mathrm{d} \boldsymbol{x}}{\mathrm{d} s}=0 .
$$


There may be circumstances where the homogenized free boundary does develop nonsmooth features (for example, computations [22] show the development of a corner). We will not address this here, but the possibility certainly warrants further investigation.

We introduce the "interior" region

$$
\Omega_{\epsilon}(t)=\left\{\boldsymbol{x} \mid \operatorname{dist}(\boldsymbol{x}, \Gamma(t ; \epsilon))>C_{3} \epsilon \ln \left(\epsilon^{-1}\right)\right\} .
$$

The estimate (18) provides a bound for $h$ and its first and second derivatives on $\Omega_{\epsilon}$ independent of $\epsilon$, whereas (10) indicates that $\Omega_{\epsilon} \rightarrow \Omega_{0}$ in the sense of boundary convergence. This suggests that the leading order approximation for $h$ is well defined on the domain $\Omega_{0}$ and independent of $\epsilon$.

Near the free boundary, we will propose a different expansion in rescaled variables. We define the set

$$
B_{\epsilon}=\left\{\boldsymbol{x} \mid \operatorname{dist}(\boldsymbol{x}, \Gamma(t))<C_{4} \epsilon \ln \left(\epsilon^{-1}\right)\right\}, \quad C_{4}>C_{3} .
$$

This definition guarantees that $B_{\epsilon}$ and $\Omega_{\epsilon}$ overlap as $\epsilon \rightarrow 0$, which allows application of matching conditions.

\subsection{Expansion in the interior}

The foregoing arguments are meant to provide heuristic motivation and plausibility for the proposed expansion

$$
h=H_{0}(\boldsymbol{x}, t)+\epsilon H_{1}(\boldsymbol{x}, t, \tau)+\mathcal{O}\left(\epsilon^{2}\right), \quad \lambda=\lambda_{0}+\mathcal{O}(\epsilon),
$$

that holds on $\Omega_{\epsilon}$ for each $\epsilon>0 . H_{0}, H_{1}$ are taken to be $C^{2}\left(\Omega_{0}\right)$ in space and $C^{1}$ in $t$ and $\tau$. The correction term $H_{1}$ is meant to capture temporal fluctuations about the mean dynamics, and is therefore taken to be bounded:

$$
\left|H_{1}\right|<C, \quad C \text { depending only on } \boldsymbol{x}, t .
$$

The leading order problem solved by $H_{0}$ is essentially the same as (2),

$$
\Delta H_{0}=\lambda_{0},\left.\quad H_{0}\right|_{\Gamma_{0}}=0, \quad \Gamma_{0} \equiv \partial \Omega_{0}, \quad \int_{\Omega_{0}} H_{0} \mathrm{~d} x=M \equiv \text { volume }
$$

Provided $\left|\nabla H_{0}\right|>0$ on the boundary, the regularity of $H_{0}$ implies that $\Gamma_{0}$ is a $C^{1}$ curve (see point 3 above). We can identify the "macroscopic" contact angle and normal direction as

$$
\theta_{M}=\left|\nabla H_{0}\right|, \quad \boldsymbol{n}_{0}=\frac{\nabla H_{0}}{\left|\nabla H_{0}\right|}, \quad \text { on } \Gamma_{0} .
$$

It should be emphasized that $\boldsymbol{n}_{0}$ is not the limit of the normals to the curve $\Gamma$ (except perhaps in a weak sense), but is rather determined by limit of the interior solutions as $\epsilon \rightarrow 0$.

The motion of $\Gamma_{0}$ can be characterized by the free boundary condition

$$
F_{\text {hom }}\left(\theta ; \boldsymbol{n}_{0}\right)=\frac{\left(H_{0}\right)_{t}}{\left|\nabla H_{0}\right|} \quad \text { when } H_{0}=0 .
$$

The dynamics of $H_{0}$ and therefore $F_{\text {hom }}$ will be ascertained by asymptotic matching and time averaging. 


\subsection{Expansion near the free boundary}

For the expansion on $B_{\epsilon}$, a local rectilinear coordinate system $(r, s)$ is used (see Fig. 1). The coordinate $s$ varies in direction tangent to a point $\boldsymbol{x}_{0} \in \Gamma_{0}$ and $r$ is in the negative normal direction so that $r>0$ is the fluid's interior. We suppose that the normal $\boldsymbol{n}_{0}$ at $\boldsymbol{x}_{0}$ is in a "rational" direction, in other words $\boldsymbol{n}_{0} \| n_{1} \mathbf{i}+n_{2} \mathbf{j}$ where $n_{1}, n_{2} \in \mathbb{Z}$. This ensures that $F$ is periodic with period $\epsilon p_{n} \equiv \epsilon \sqrt{n_{1}^{2}+n_{2}^{2}}$ in $r$ and $s$.

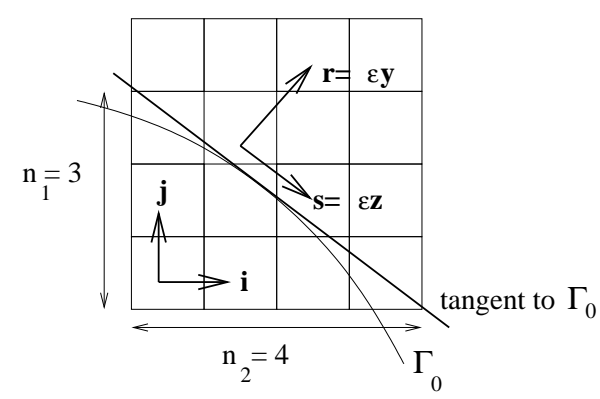

FIG. 1. Coordinate system used for the inner expansion $\left(\boldsymbol{n}_{0}=3 \mathbf{i}+4 \mathbf{j}\right.$ here $)$.

We argue that it is sufficient to define $F_{\text {hom }}(\theta ; \boldsymbol{n})$ only for rational normal directions. For an irrational normal $\boldsymbol{n}(\boldsymbol{x})$ to the curve at a point $\boldsymbol{x}$ on $\Gamma_{0}$, we can find a sequence $\boldsymbol{x}_{i} \rightarrow \boldsymbol{x}, \boldsymbol{x}_{i} \in \Gamma_{0}$, where $\boldsymbol{n}\left(\boldsymbol{x}_{i}\right)$ are rational directions. Since $H_{0}$ is continuously differentiable in space and time, using 25] we have

$$
\frac{\left(H_{0}\right)_{t}}{\left|\nabla H_{0}\right|}(\boldsymbol{x})=\lim _{i \rightarrow \infty} \frac{\left(H_{0}\right)_{t}}{\left|\nabla H_{0}\right|}\left(\boldsymbol{x}_{i}\right)=\lim _{i \rightarrow \infty} F_{\mathrm{hom}}\left(\theta_{M}\left(\boldsymbol{x}_{i}\right) ; \boldsymbol{n}_{i}\right) .
$$

In other words, the homogenized velocity only needs to be computed on a dense set of $\Gamma_{0}$ to entirely specify the leading order dynamics.

The expansion we propose near the contact line is

$$
h=\epsilon h_{1}(y, z, \tau, t)+\mathcal{O}\left(\epsilon^{2}\right), \quad y=\epsilon^{-1} r, z=\epsilon^{-1} s,
$$

where $h_{1}$ is $C^{2}$ on the domain $\left\{h_{1}>0\right\} \cap[-\infty, \infty] \times\left[0, p_{n}\right], p_{n}$-periodic in $z$, and $C^{1}$ in time. This is assumed valid on each local subregion of $B_{\epsilon}$ for each $\epsilon>0$.

The moving boundary problem (2), (5) written in the inner coordinate system is therefore to leading order

$$
\begin{aligned}
\Delta h_{1} & =0 \quad \text { for } h_{1}>0, \\
\left(h_{1}\right)_{\tau} & =F\left(\left|\nabla h_{1}\right| ; y, z\right)\left|\nabla h_{1}\right| \quad \text { on } \Gamma_{1},
\end{aligned}
$$

where $\Gamma_{1}=\partial\left\{h_{1}>0\right\}$. By construction, the velocity law is $p_{n}$-periodic in $y$ and $z$ and it depends on the leading order normal direction $\boldsymbol{n}_{0}$ :

$$
F\left(\theta ; y+k_{1} p_{n}, z+k_{2} p_{n}\right)=F(\theta ; y, z) \quad \text { for all }\left(k_{1}, k_{2}\right) \in \mathbb{Z}^{2} .
$$

The Laplace problem in 28 is to be solved in a simply connected subset of the channel $(-\infty, \infty) \times$ $\left[0, p_{n}\right]$ which extends to $y=+\infty$ (see Fig. 2). The boundary conditions on $h_{1}$ are

$$
h_{1}(z, y, \tau)=h\left(z+p_{n}, y, \tau\right) \text {, }
$$




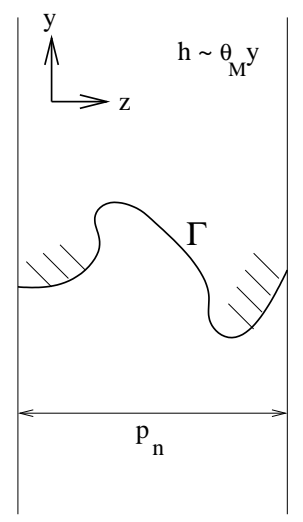

FIG. 2. Diagram of the free boundary "cell" problem.

$$
\lim _{y \rightarrow \infty} \frac{\partial h_{1}}{\partial y}=-\frac{\partial H_{0}}{\partial \boldsymbol{n}_{0}}=\theta_{M} .
$$

The first of these arises from the assumption of periodicity in $z$. The far field boundary condition comes from asymptotic matching, by equating the expansions (21) and (27) and taking $\epsilon \rightarrow 0$ and $y \rightarrow \infty$ simultaneously.

\subsection{Matching and time averaging}

To complete the analysis, the dynamics of the interior and boundary solutions need to be connected. Computing $\partial h / \partial t$ for both expansions and equating gives

$$
\frac{\partial h_{1}}{\partial \tau}=\left(\frac{\partial H_{0}}{\partial t}+\frac{\partial H_{1}}{\partial \tau}\right)+\mathcal{O}(\epsilon)
$$

which, for each $\epsilon>0$, holds on the overlapping region $\Omega_{\epsilon} \cap B_{\epsilon}$. We can now average over the fast scale by applying $T^{-1} \int_{0}^{T} \mathrm{~d} \tau$ to equation 33 :

$$
T^{-1} \int_{0}^{T} \frac{\partial h_{1}}{\partial \tau} \mathrm{d} \tau=\frac{\partial H_{0}}{\partial t}+\left.T^{-1} H_{1}\right|_{t=0} ^{t=T}+\mathcal{O}(\epsilon) .
$$

We conclude by taking the limits $\epsilon \rightarrow 0, T \rightarrow \infty, y \rightarrow \infty$ in such a way that $T \epsilon \rightarrow 0$ and $y \epsilon \rightarrow 0$. Using 22, yields

$$
\left.\frac{\partial H_{0}}{\partial t}\right|_{\Gamma_{0}}=\lim _{T \rightarrow \infty, y \rightarrow \infty} T^{-1} \int_{0}^{T} \frac{\partial h_{1}}{\partial \tau} \mathrm{d} \tau .
$$

To summarize, the homogenized velocity is obtained by solving the free boundary problem 28, (31), (32) and using (25) and (35) to give

$$
F_{\text {hom }}(\theta ; \boldsymbol{n})=\theta_{M}^{-1} \lim _{T \rightarrow \infty, y \rightarrow \infty} T^{-1} \int_{0}^{T} \frac{\partial h_{1}}{\partial \tau} \mathrm{d} \tau .
$$




\section{A comparison principle}

The main tool for investigating the limit $\sqrt{36}$ is a comparison principle similar to the one used by Kim [36] to construct viscosity solutions of Hele-Shaw and Stefan problems. We focus here only on smooth solutions, which can be expected for mild heterogeneities. This makes the analysis considerably more transparent, although similar results should hold for nonsmooth solutions provided they obey the same comparison principle.

Following [36], we define notions of super- and subsolutions.

Definition 1 Suppose that $h(y, z, t)$ is a smooth function, $p_{n}$-periodic in $z$ and $\{h>0\}$ is a simply connected subset of $(-\infty, \infty) \times[0, p]$. Let $\Gamma(t)=\{h=0\}$ be a smooth, nonintersecting curve which is differentiable in time (in the sense that it admits a differentiable parameterization). Then $h$ is a supersolution (respectively subsolution) if

1. $\Delta h \leqslant(\geqslant) 0$ on the set $\{h>0\}$.

2. There exists $y^{*}$ so that $h(y, z)>0$ when $y>y^{*}$ and $\lim _{y \rightarrow \infty} \partial h / \partial y \geqslant(\leqslant) \theta_{M}$.

3. $d \Gamma / d t \geqslant(\leqslant) F(-\partial h / \partial \boldsymbol{n} ; \boldsymbol{x})$ for $x \in \Gamma$.

We also have a notion of comparing two functions (typically super- or subsolutions).

DEFINITION 2 Suppose that $h_{1}, h_{2}$ are smooth functions, $p$-periodic in $z$ and $\left\{h_{1,2}>0\right\}=\Omega_{1,2}(t)$ are simply connected subsets of $(-\infty, \infty) \times[0, p]$. Then if $\Omega_{1}(t)$ is a proper subset of $\Omega_{2}(t)$, we say that $h_{1}$ and $h_{2}$ are separated at time $t$, and write $h_{1} \prec h_{2}$.

It is convenient to rephrase the Laplace problem (28) by introducing the change of variables $w=h_{1}-\theta_{M} y$ so that we have the boundary conditions

$$
\left.w\right|_{\Gamma_{1}}=-\theta_{M} y, \quad \lim _{y \rightarrow \infty} w(y, z)=w_{\infty} .
$$

Note that the homogenized velocity can be written as

$$
F_{\text {hom }}(\theta ; \boldsymbol{n})=\theta_{M}^{-1} \lim _{T \rightarrow \infty} \frac{1}{T} \int_{0}^{T} \frac{\mathrm{d} w_{\infty}}{\mathrm{d} t} \mathrm{~d} t .
$$

The far field value $w_{\infty}$ should be regarded as an unknown of the problem, and can be bounded by the values $w$ takes on $\Gamma_{1}$ :

PROPOSITION 1 (i) If $w$ solves $\Delta w=0$ on $\Omega(t)$ with boundary conditions (37),

$$
\min _{\Gamma_{1}} w \leqslant w_{\infty} \leqslant \max _{\Gamma_{1}} w .
$$

(ii) Let $h_{1}$ and $h_{2}$ be sub- and supersolutions, respectively. If $h_{1} \prec h_{2}$ then for the corresponding functions $w_{1,2}=h_{1,2}-\theta_{M} y$ we have

$$
\lim _{y \rightarrow \infty} w_{1} \leqslant \lim _{y \rightarrow \infty} w_{2} .
$$

Proof. For (i) the upper bound is obtained by setting $g=w-\max _{\Gamma_{1}} w$ and applying the PhragménLindelöf principle ([44, p. 97]), which gives $g \leqslant 0$ in $\Omega_{\epsilon}$. The lower bound is similar.

For (ii), we observe that $w_{2}+\theta_{M} y$ takes on nonnegative boundary values on any finite domain $\left\{h_{2}>0\right\} \cap\left\{y<y^{*}\right\}$, so it follows that $w_{2} \geqslant-\theta_{M} y$ and $w_{2} \geqslant w_{1}$ on $\left\{h_{1}=0\right\}$. The PhragménLindelöf principle applied to $w_{2}-w_{1}$ on the domain $\Omega_{1}$ implies $w_{1}>w_{2}$ in $h_{1}>0$. 
We can now establish the main tool for analysis of the cell problem.

PROPOSITION 2 (Comparison principle) Let $h_{1}$ and $h_{2}$ be sub- and supersolutions, respectively, and suppose that $h_{1} \prec h_{2}$ at time $t=0$. Then either $h_{1}=h_{2}$ after some positive time or $h_{1} \prec h_{2}$ for all $t>0$.

Proof. Set $\Omega_{1,2}=\left\{h_{1,2}>0\right\}$ and let $\Gamma_{1,2}$ be the respective boundaries. If $\Omega_{1}(t) \subset \Omega_{2}(t)$ does not hold for all $t \geqslant 0$, then there exists a maximal time $T$ for which this happens, and a contact point $P \in \Gamma_{1,2}(T)$. It follows that the normal velocity of $\Gamma_{1}$ is greater than or equal to that of $\Gamma_{2}$ at $P$. The monotonicity of the velocity function $F$ implies that at $P$,

$$
\frac{\partial h_{1}}{\partial n} \leqslant \frac{\partial h_{2}}{\partial n} .
$$

The difference $h=h_{1}-h_{2}$ satisfies Laplace's equation with the boundary condition $h=-h_{2} \leqslant 0$ on $\Gamma_{1}$. If $h \equiv 0$ on $\Gamma_{1}$, then uniqueness implies that both solutions will remain identical for $t>T$. If not, Proposition 1 implies there is a large $y^{*}$ so that $h(y, z)<0$ if $y>y^{*}$. Then $h$ must attain its maximum on the boundary of the bounded domain $D=\Omega_{1} \cup\left\{y<y^{*}\right\}$. This maximum cannot occur on the periodic boundaries $\left(z=0\right.$ or $\left.z=p_{n}\right)$ of $D$. If it did, one could simply shift the domain in $z$ so that the maximum point was in the interior. A maximum therefore occurs at the contact point $P$. Because of the assumed smoothness of the boundary of $D$, the Hopf maximum principle ([44, p. 65]) shows that $\partial h / \partial n$ is strictly positive at the contact point $P$, which contradicts [39].

\section{The homogenized front velocity}

This section establishes the existence of the limit in (36) and explores some of its properties. The following assumptions are placed on $F(\theta ; \boldsymbol{x})$ to get control over the front width:

(1) $F(\theta ; \boldsymbol{x})$ is continuous, strictly increasing in $\theta$.

(2) For any $\theta_{M}>0$, there exist constants $C_{1}, C_{2}, C_{3}>0$ so that

$$
\mu-C_{3}+C_{1} \theta \leqslant F(\theta ; \boldsymbol{x}) \leqslant C_{2}\left(\frac{\theta-\theta_{M}}{\theta_{M}}\right), \quad \mu=\min _{\boldsymbol{x}} F\left(\theta_{M}, \boldsymbol{x}\right) .
$$

The second condition ensures that (A) the front velocity will get large for steep contact angles and (B) the front recedes fast enough for shallow contact angles. Using (40), control over the front width can be obtained:

Proposition 3 (Bound on front width) Suppose that $h(y, z, t), \Gamma_{1}(t)=\{h=0\}$ is a smooth, non-self-intersecting solution of (28)-(32). Let $W=\max _{\Gamma} y-\min _{\Gamma} y$. If the front velocity satisfies the conditions 40 there exists an upper bound $B$ so that if $W(0) \leqslant B$, then $W(t) \leqslant B$ for all $t \geqslant 0$.

The proof of this is deferred to the Appendix.

We can now prove the main result of this section, which establishes the existence of the limit in 36.

THEOREM 1 Suppose that $h(y, z, t), \Gamma_{1}(t)=\{h=0\}$ is a smooth, non-self-intersecting solution of (28)-(32), whose initial front $\Gamma_{1}(0)$ has width smaller than the bound guaranteed by Proposition 3 Then 
- The following limit exists (i.e. the long time average front velocity):

$$
F_{\text {hom }}\left(\theta_{M} ; \boldsymbol{n}\right)=\theta_{M}^{-1} \lim _{T \rightarrow \infty} \frac{1}{T} \int_{0}^{T} \frac{\mathrm{d} w_{\infty}}{\mathrm{d} t} \mathrm{~d} t .
$$

- The limit is unique: $F_{\mathrm{hom}}$ is independent of the initial condition.

- $F_{\text {hom }}\left(\theta_{M} ; \boldsymbol{n}\right)$ is nondecreasing in $\theta_{M}$.

Proof. Existence of the limit. For a solution $h=w+\theta_{M} y$ define the channel-averaged interface position and average velocity as

$$
\bar{y}(T)=\frac{1}{p_{n}} \int_{\Gamma_{1}(T)} y \mathrm{~d} z, \quad v(T) \equiv \frac{\bar{y}(0)-\bar{y}(T)}{T} .
$$

Proposition 1 and the boundary conditions 37 imply that

$$
\bar{y}(T)-B \leqslant-\theta_{M}^{-1} w_{\infty}(T) \leqslant \bar{y}(T)+B
$$

where $B$ is an upper bound on front width. Dividing by $T$, integrating and taking $T \rightarrow \infty$ we have

$$
\lim _{T \rightarrow \infty} \frac{\bar{y}(0)-\bar{y}(T)-B}{T} \leqslant \theta_{M}^{-1} \lim _{T \rightarrow \infty} \frac{1}{T} \int_{0}^{T} \frac{\mathrm{d} w_{\infty}}{\mathrm{d} t} \mathrm{~d} t \leqslant \lim _{T \rightarrow \infty} \frac{\bar{y}(0)-\bar{y}(T)+B}{T},
$$

which implies the equality of limits

$$
\lim _{T \rightarrow \infty} v(T)=\theta_{M}^{-1} \lim _{T \rightarrow \infty} \frac{1}{T} \int_{0}^{T} \frac{\mathrm{d} w_{\infty}}{\mathrm{d} t} \mathrm{~d} t .
$$

Since the front velocity $F(\theta ; \boldsymbol{x})$ is bounded from below, we can define

$$
v^{*}=\liminf _{T \rightarrow \infty} v(T) .
$$

For any $\delta>0$, there is a time $T_{\delta}$ so that

$$
v\left(T_{\delta}\right) \leqslant v^{*}+\delta
$$

Let $M$ be a positive integer so that $\bar{y}\left(T_{\delta}\right)+B \leqslant M p_{n}$. By periodicity, $h_{1}=h\left(y-M p_{n}, z, T-T_{\delta}\right)$ is also a solution. Proposition 2 yields $h \prec h_{1}$ for all $T>T_{\delta}$. It follows that the position of front $\Gamma_{1}$ has the bound

$$
\bar{y}(T)-\bar{y}(0) \leqslant n\left(\bar{y}\left(T_{\delta}\right)-\bar{y}(0)\right)+B+p_{n}, \quad(n-1) T_{\delta} \leqslant T \leqslant n T_{\delta},
$$

for any $n \in \mathbb{Z}^{+}$. Dividing by $T$ and using 42 and 44 gives

$$
v(T) \leqslant \frac{n}{n-1}\left(v^{*}+\delta\right)+\frac{B+p_{n}}{(n-1) T_{\delta}} .
$$

This implies that for any $\delta>0$,

$$
\limsup _{T \rightarrow \infty} v(T) \leqslant v^{*}+\delta
$$

which shows that $\lim _{T \rightarrow \infty} v(T)=v^{*}$. 
Uniqueness of the limit. Suppose that $h_{1}$ and $h_{2}$ are solutions and $v_{1}(T), \bar{y}_{1}(T)$, etc. are average velocities and front positions. We can ensure that $h_{1} \prec h_{2}$ initially by a suitable periodic translation which does not affect the long time average (41). Since $h_{1} \prec h_{2}$ for all time, it follows that for large $T$,

$$
v_{1}(T)=\frac{\bar{y}_{1}(0)-\bar{y}_{1}(T)}{T} \leqslant \frac{\bar{y}_{2}(0)-\bar{y}_{2}(T)}{T}+\frac{\bar{y}_{1}(0)-\bar{y}_{2}(0)}{T} \leqslant v_{2}(T)+\mathcal{O}\left(T^{-1}\right) .
$$

Similarly, by a suitable periodic translation we could ensure that $h_{1}(t) \prec h_{2}(t)$ so that $v_{2}(T) \leqslant$ $v_{1}(T)+\mathcal{O}\left(T^{-1}\right)$. It follows that the long time limits must be equal.

Monotonicity. Suppose that $h_{1}$ is any solution corresponding to $\theta_{M}=\theta_{1}$ with initially bounded front width. Let $h_{2}$ be a solution corresponding to $\theta_{M}=\theta_{2}>\theta_{1}$. We can guarantee that $h_{1} \prec h_{2}$ at time $t=0$ by a suitable periodic translation in $y$. With respect to $\Theta_{M}=\theta_{1}, h_{2}$ is a supersolution, so $h_{1} \prec h_{2}$ for all time $t>0$. It follows that $F_{\text {hom }}\left(\theta_{1} ; \boldsymbol{n}\right) \leqslant F_{\text {hom }}\left(\theta_{2} ; \boldsymbol{n}\right)$.

\section{Examples and a numerical method}

This section demonstrates the practical consequences of the foregoing theory. In general, one must resort to numerical computation to compute the homogenized velocity. We will formulate the problem by an efficient integral equation algorithm and demonstrate the feasibility of this approach by means of computational examples. We begin with a simple example where an explicit formula can be found.

\subsection{A simple example: one-dimensional motion}

The simplest situation is where $F(\theta ; y, z)$ has no $z$-dependence. In this case, it is sufficient to consider exact planar solutions of the form

$$
h=\theta_{M}(y-Y(t)) .
$$

It follows that

$$
\frac{\mathrm{d} Y}{\mathrm{~d} t}=-F\left(\theta_{M} ; Y(t)\right)
$$

If $F\left(\theta_{M} ; y\right)$ is of one sign, periodic motion will ensue. If $T$ is the period of this oscillating solution then

$$
T=\int_{0}^{p_{n}}\left(\frac{\mathrm{d} Y}{\mathrm{~d} t}\right)^{-1} \mathrm{~d} y
$$

from which one finds the average velocity as

$$
F_{\text {hom }}\left(\theta_{M}\right)=\frac{p_{n}}{\int_{0}^{p_{n}} F\left(\theta_{M} ; y\right)^{-1} \mathrm{~d} y} .
$$

If the integral diverges, the front is pinned and we take $F_{\text {hom }}$ to be 0 .

\subsection{Integral equation formulation}

The problem (28)-(32), (37) can be written as a pair of integral equations to expedite numerical simulation of the cell problem and computation of $F_{\text {hom }}$. The Green's function appropriate for an 
infinite channel with periodic boundary conditions (see also [31]) is

$$
G(y, z)=\frac{1}{4 \pi} \ln \left[\sin ^{2}\left(\frac{\pi z}{p_{n}}\right) \cosh ^{2}\left(\frac{\pi y}{p_{n}}\right)+\cos ^{2}\left(\frac{\pi z}{p_{n}}\right) \sinh ^{2}\left(\frac{\pi y}{p_{n}}\right)\right] .
$$

Taking the inner product with $\Delta w=0$ on the domain $\Omega_{\epsilon} \cap\left\{y<y^{*}\right\}$ gives the standard integral representation

$$
\begin{array}{r}
\int_{\Gamma_{1} \cup\left\{y=y^{*}\right\}}\left(w(y, z) \frac{\partial G}{\partial n}\left(y-y_{0}, z-z_{0}\right)-G\left(y-y_{0}, z-z_{0}\right) \frac{\partial w}{\partial n}(y, z)\right) \mathrm{d} s(y, z) \\
= \begin{cases}w\left(y_{0}, z_{0}\right), & \left(y_{0}, z_{0}\right) \in \operatorname{int} \Omega_{\epsilon}, \\
\frac{1}{2} w\left(y_{0}, z_{0}\right), & \left(y_{0}, z_{0}\right) \in \Gamma_{1},\end{cases}
\end{array}
$$

where $n$ is the outward normal.

As $y \rightarrow \infty$, we have the asymptotics $G \sim y / 2 p_{n}+o(1)$ and $w \sim w_{\infty}+o(1)$. Taking $y^{*} \rightarrow \infty$ in (51) leads to

$$
\begin{aligned}
& \frac{w\left(y_{0}, z_{0}\right)-w_{\infty}}{2}=\int_{\Gamma_{1}}\left(w(y, z) \frac{\partial G}{\partial n}\left(y-y_{0}, z-z_{0}\right)\right. \\
& \left.\quad-G\left(y-y_{0}, z-z_{0}\right) \frac{\partial w}{\partial n}(y, z)\right) \mathrm{d} s(y, z), \quad\left(y_{0}, z_{0}\right) \in \Gamma_{1} .
\end{aligned}
$$

A similar calculation taking $y^{*} \rightarrow \infty$ and then $y_{0} \rightarrow \infty$ gives another equation

$$
w_{\infty}=\frac{1}{p_{n}} \int_{\Gamma_{1}}\left(y \frac{\partial w}{\partial n}-w n_{y}\right) \mathrm{d} s,
$$

where $n_{y}$ is the $y$ component of the normal vector. Equations $52--(53)$ form a system to be solved for unknowns $\partial w / \partial \boldsymbol{n}, w_{\infty}$. This allows the contact angle $\theta=-\partial w / \partial \boldsymbol{n}-\theta_{M} n_{y}$ and the associated normal interface velocity to be computed. Other details concerning the discretization of integrals and the time stepping procedure have been reported elsewhere [22].

\subsection{Example: array of large defects}

The practical consequences of the theory which has been developed can now be illustrated. A simple nonuniform velocity law can be constructed using the Cox-Voinov law (6) where the equilibrium contact angle $\theta_{e}$ has spatial dependence. Assuming that the $(y, z)$ coordinates are aligned with the underlying periodicity, this example supposes

$$
\theta_{e}= \begin{cases}.6, & (z-\pi)^{2}+(y-\pi)^{2}<1.5^{2} \\ .3, & \text { otherwise }\end{cases}
$$

With this orientation the (rescaled) spatial periodicity is taken as $p_{n}=2 \pi$. To investigate anisotropy, we also conducted simulations where the orientation was rotated by $45^{\circ}$ and the corresponding spatial periodicity was $2 \sqrt{2} \pi$. This particular example is meant to model a flow over a rectangular array of circular "defects"-regions which are less wetting than the rest of the solid surface. All 


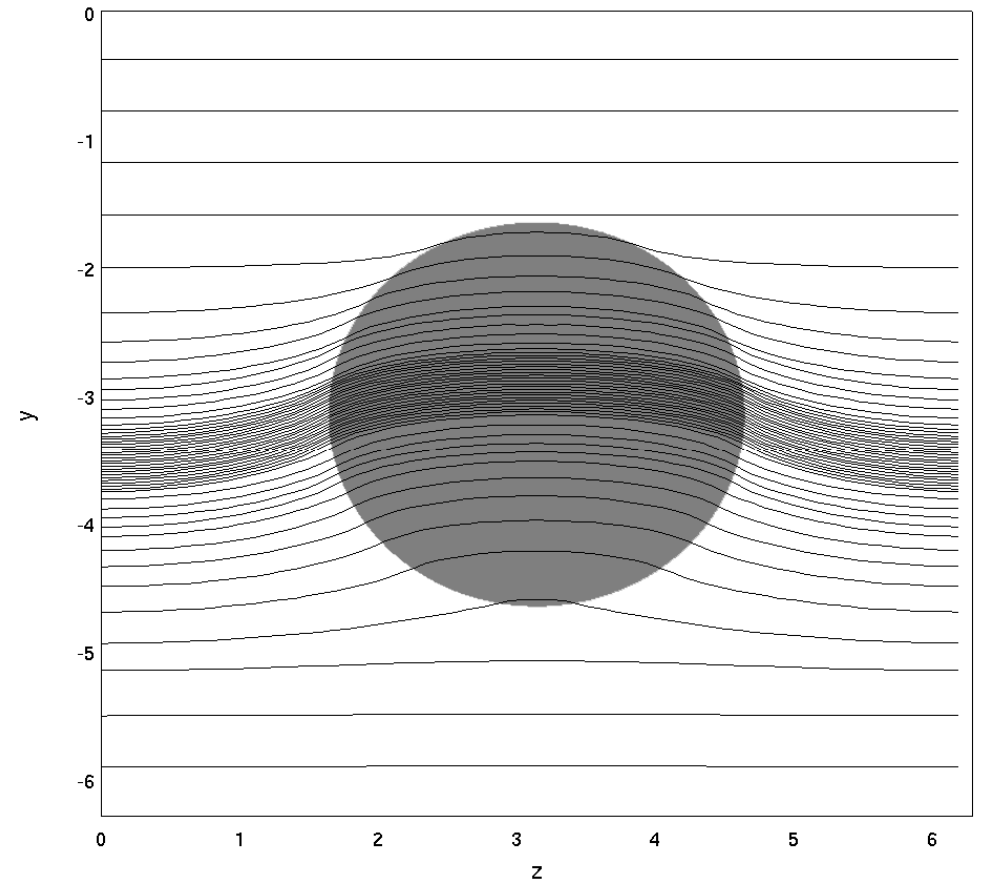

FIG. 3. Front motion when $\theta_{M}=.48$, slightly above the pinning threshold. The $y$ coordinate is aligned with the underlying spatial periodicity in this example. The shaded regions are where the contact angle is higher (less wetting). Contours are roughly spaced in equal time intervals.

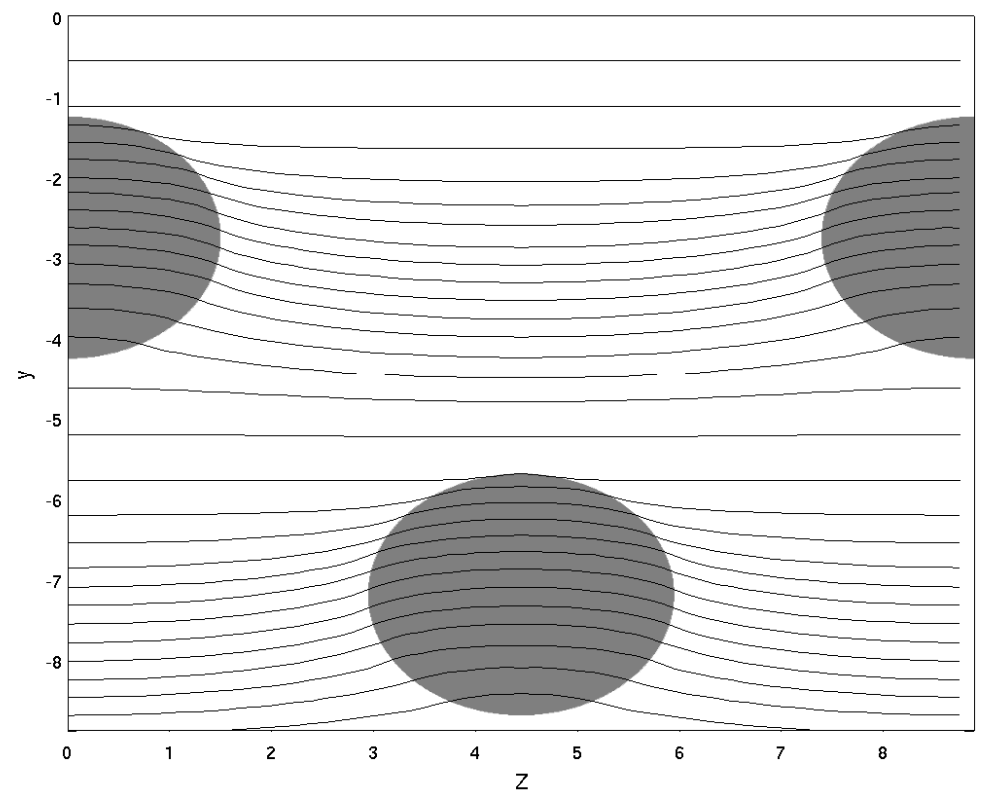

FIG. 4. Front motion when the $y$ coordinate is oriented at a $45^{\circ}$ angle to the underlying spatial periodicity $\left(\theta_{m}=0.48\right)$. 


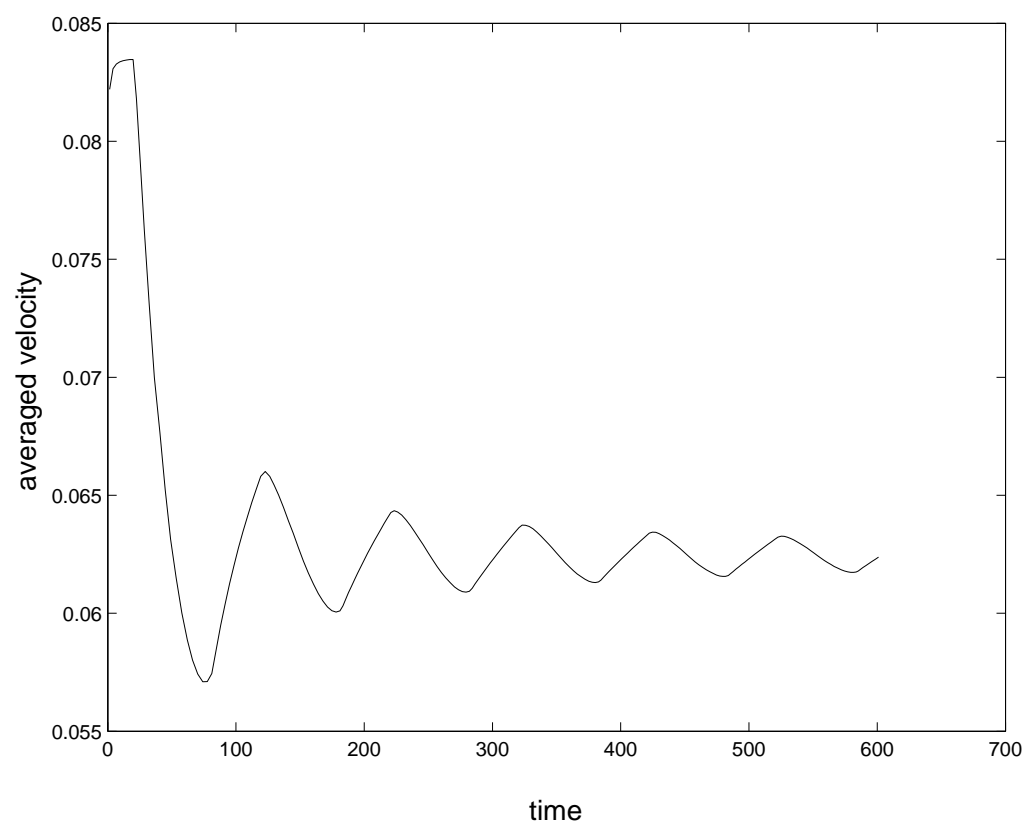

FIG. 5. Time averaged front velocity as a function of time $\left(\theta_{m}=.48\right)$.

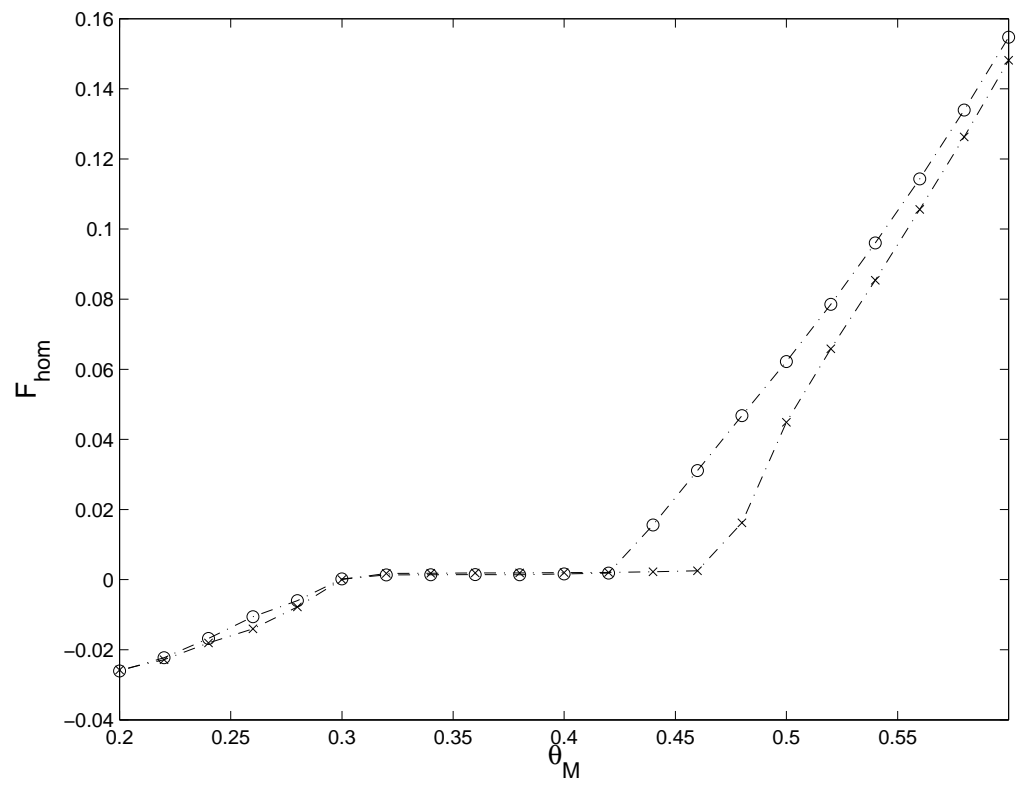

FIG. 6. Homogenized front velocities. The x's denote computations for orientation angle $0^{\circ}$ and the circles for orientation angle $45^{\circ}$. 
computations used initial data that was just a flat interface $y=0$, although the uniqueness of the homogenized velocity should mean that this choice is more or less inconsequential.

Figure 3 shows typical front evolution when the normal to the macroscopic front is aligned with the array. In Figure 4 the normal is instead at a $45^{\circ}$ angle to the array. The time averaged front motion (see (42) typically oscillates and converges (somewhat slowly) to a long time limit (Figure 5). Nonlinear regression was used to extrapolate this limit to avoid unnecessarily lengthy computation.

The long time averaged velocities $F\left(\theta_{M} ; \boldsymbol{n}\right)$ were computed for many values of $\theta_{M}$ for both orientations (Figure 6). Over a closed interval of $\theta_{M}$, the front is pinned and the averaged velocity is zero. The endpoints of this interval can be thought of as the receding and advancing contact angles. Anisotropy is present in both the overall velocities and in the interval on which pinning occurs. Experiments on periodically structured surfaces have also found this [12, 24].

\subsection{Example: small randomly placed defects}

As a second more elaborate example, we consider smaller randomly placed defects (still arranged periodically), with the same velocity law (6). Figure 7] shows typical front motion. The homogenized front speed (Figure 8 ) was computed as before. The pinning interval is markedly smaller than the case with larger defects. The average front motion is, on the other hand, quantitatively similar.

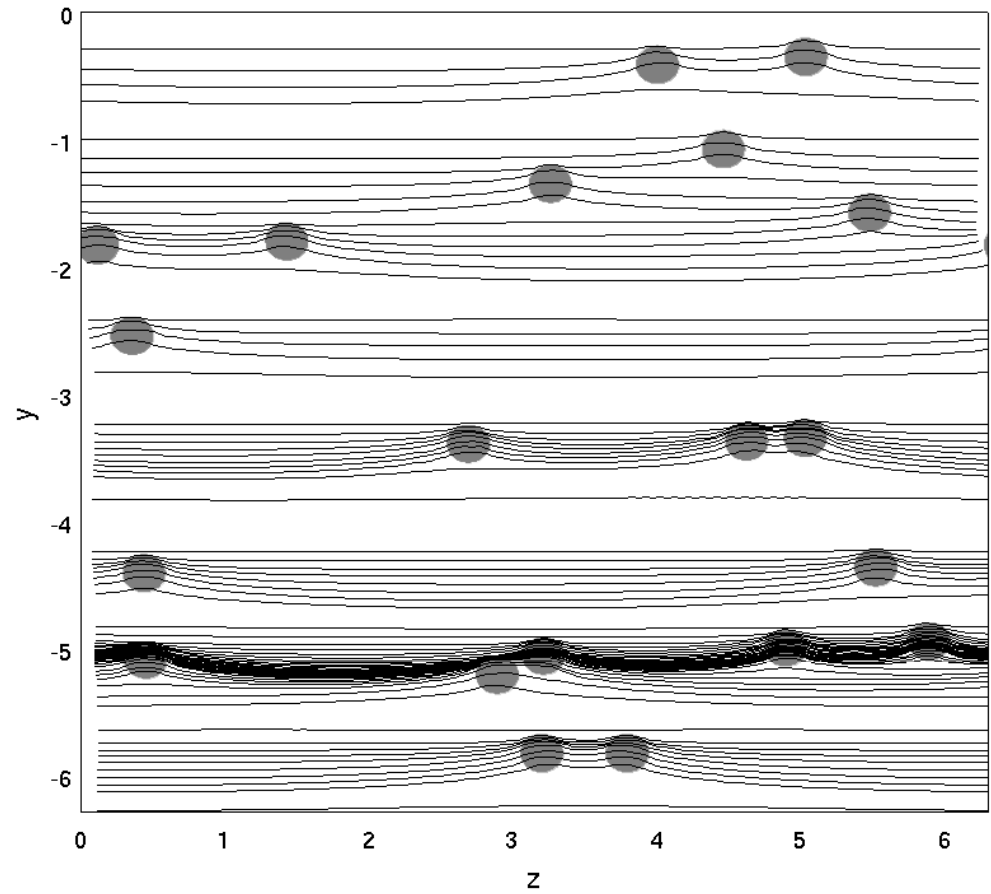

FIG. 7. Front motion for $\theta_{m}=0.5$. There were 20 defects of radius 0.15 . Slowing of the front around defects is modified by the lateral spacing. 


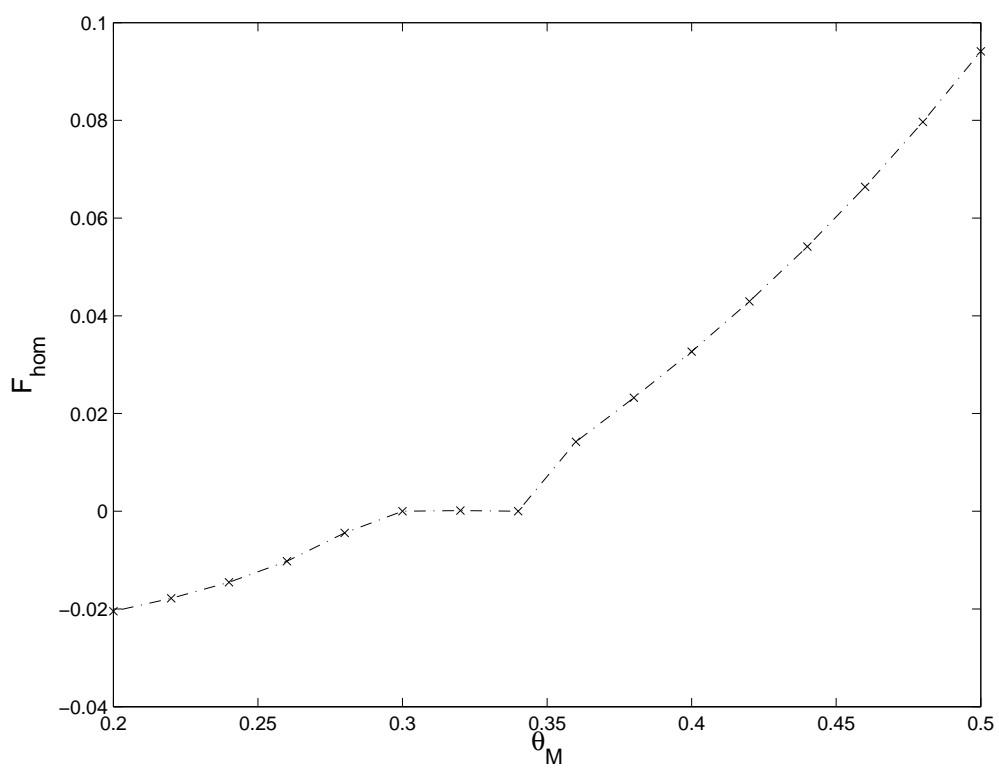

FIG. 8. Homogenized front velocities for the case of many defects. The x's denote actual computations, and the dashed line is an interpolation.

\section{Appendix: proof of bound on front width}

The proof relies on comparison functions which control the normal derivatives at extreme points on $\Gamma$. Constants $C, \beta$ are generic and assumed independent of $W$.

Proof. Let $\left(x_{m}, y_{m}\right),\left(x_{M}, y_{M}\right) \in \Gamma$ be points on the interface where the minimum and maximum, respectively, of $y$ is achieved. Then $w$ is harmonic in $[0, p] \times\left[y_{M},+\infty\right)$ and has a Green's function representation

$$
w\left(x_{0}, y_{0}\right)-w_{\infty}=\int_{0}^{p} G_{0}\left(x, y_{M}, x_{0}, y_{0}\right)\left(w\left(x, y_{M}\right)-w_{\infty}\right) \mathrm{d} x, \quad y_{0}>y_{M},
$$

where $G_{0}=G\left(x, y, x_{0}, y_{0}\right)-G\left(x,-y, x_{0}, y_{0}\right)$. It is easily verified that $G_{0}$ satisfies a bound of the form $\left\|G_{0}\right\|_{L^{1}(x)}<C \exp (-\beta y)$, so it follows that $w$ satisfies a pointwise estimate

$$
\begin{aligned}
\left|w(x, y)-w_{\infty}\right| & \leqslant C\left\|w\left(x, y_{M}\right)-w_{\infty}\right\|_{L^{\infty}} \exp \left(-\beta\left[y-y_{M}\right]\right) \\
& \leqslant C \theta_{M} W \exp \left(-\beta\left[y-y_{M}\right]\right) .
\end{aligned}
$$

In the last step the maximum principle was used to obtain $\left|w-w_{\infty}\right| \leqslant \max \left(\left|w_{\infty}+\theta y_{m}\right|\right.$, $\left.\left|w_{\infty}+\theta y_{M}\right|\right) \leqslant W \theta_{M}$.

We now construct the linear comparison functions

$$
\begin{aligned}
& u_{1}=-\theta_{M} y_{m}+s_{1}\left(y-y_{m}\right), \quad s_{1}(L)=\frac{C \theta_{M} W \exp \left(-\beta\left[L-y_{M}\right]\right)+\theta_{m} y_{m}+w_{\infty}}{L-y_{m}}, \\
& u_{2}=-\theta_{M} y_{M}+s_{2}\left(y-y_{M}\right), \quad s_{2}(L)=\frac{-C \theta_{M} W \exp \left(-\beta\left[L-y_{M}\right]\right)+\theta_{M} y_{m}+w_{\infty}}{L-y_{M}},
\end{aligned}
$$


for some parameter $L$ which will be determined. By the estimate (55), the maximum principle implies that $u_{2} \leqslant w \leqslant u_{1}$ on the domain $\Omega \cup[0, p] \times\left[y_{m}, L\right]$. In particular, $w=u_{1}$ at $\left(x_{m}, y_{m}\right)$ and $w=u_{2}$ at $\left(x_{M}, y_{M}\right)$ so that the normal derivatives at the extreme points satisfy

$$
\begin{array}{rlrl}
\frac{\partial w}{\partial y}\left(x_{m}, y_{m}\right) & \leqslant s_{1}(L), & L>y_{M}, \\
\frac{\partial w}{\partial y}\left(x_{M}, y_{M}\right) \geqslant s_{2}(L), & L>0 .
\end{array}
$$

To make the rest more attractive, introduce $\rho=\left(y_{M}+w_{\infty} / \theta_{M}\right) / W$. The maximum principle implies $-\theta_{M} y_{M} \leqslant w_{\infty} \leqslant-\theta_{M} y_{m}$ so that $0 \leqslant \rho \leqslant 1$.

For the first estimate, set $\lambda=\left(L-y_{m}\right) / W$ so that

$$
\frac{\partial w}{\partial y}\left(x_{m}, y_{m}\right) \leqslant \theta_{M} \frac{C \exp (-\beta W[\lambda-1])+\rho-1}{\lambda}, \quad \lambda>0 .
$$

Now choose $\lambda$ so that $C \exp (-\beta W[\lambda-1])=1 / W$, which gives

$$
\frac{\partial w}{\partial y}\left(x_{m}, y_{m}\right) \leqslant \theta_{M} \frac{1 / W+\rho-1}{1+\ln (C W) /(\beta W)} \leqslant \theta_{M}(1 / W+\rho-1)
$$

for large enough $W$. For the second estimate, set $\lambda=\left(L-y_{M}\right) / W$ and obtain

$$
\frac{\partial w}{\partial y}\left(x_{M}, y_{M}\right) \geqslant \theta_{M} \frac{\rho-C \exp (-\beta W \lambda)}{\lambda}, \quad \lambda>0 .
$$

Choosing $\lambda$ so that $C \exp (-\beta W \lambda)=1 / W$ gives

$$
\frac{\partial w}{\partial y}\left(x_{m}, y_{m}\right) \geqslant \theta_{M} \frac{W \rho-1}{\ln (C W) / \beta} .
$$

Finally, (61), 63) and (40) are combined to give a bound on $\mathrm{d} W / \mathrm{d} t$ :

$$
\begin{aligned}
\frac{\mathrm{d} W}{\mathrm{~d} t} & =f\left(\theta_{M}+\frac{\mathrm{d} w}{\mathrm{~d} y}\left(x_{m}, y_{m}\right), x_{m}, y_{m}\right)-f\left(\theta_{M}+\frac{\mathrm{d} w}{\mathrm{~d} y}\left(x_{M}, y_{M}\right), x_{M}, y_{M}\right) \\
& \leqslant C_{1} \theta_{M}(1 / W+\rho)-C_{3}+C_{2} \frac{1-W \rho}{\ln (C W) / \beta} .
\end{aligned}
$$

For large enough $W$, this expression is negative for all $\rho \in[0,1]$.

\section{Acknowledgments}

The author acknowledges useful discussions with Inwon Kim. This work was supported by NSF award DMS-0405596.

\section{REFERENCES}

1. Alberti, G., \& DeSimone, A. Wetting of rough surfaces: a homogenization approach. Proc. R. Soc. Lond. Ser. A Math. Phys. Eng. Sci. 461 (2005), 79-97. MR 2124194 
2. Ben Amar, M., Cummings, L., \& Pomeau, Y. Points singuliers d'une ligne de contact mobile. $C$. $R$. Acad. Sci. Paris 329 (2001), 277-282. Zbl 1032.76015

3. Bico, J., Tordeux, C., \& Quere, D. Rough wetting. Europhys. Lett. 55 (2001), 214-220.

4. Brochard-Wyart, F., Quere, D., \& De Gennes, P.-G. Drops, Bubbles, Pearls, Waves: the World of Capillarity. Springer, New York (2003).

5. Caffarelli, L. A., Lee, K.-A., \& Mellet, A. Singular limit and homogenization for flame propagation in periodic excitable media. Arch. Ration. Mech. Anal. 172 (2004), 153-190. Zbl 1058.76070 MR 2058162

6. CAMERon, A. Principles of Lubrication. Longmans, London (1966).

7. CASsie, A. B. D., \& BAXTER, S. Wettability of porous surfaces. Trans. Faraday Soc. 40 (1944), 546551.

8. Cox, R. G. The dynamics of the spreading of liquids on a solid surface. Part 1. Viscous flow. J. Fluid Mech. 168 (1986), 169-194. Zbl 0597.76102

9. CRACiUn, B., \& Bhattacharya, K. Effective motion of a curvature-sensitive interface through a heterogeneous medium. Interfaces Free Bound. 6 (2004), 151-173. Zbl 1061.35148 MR 2079601

10. Crandall, M. G., \& Lions, P.-L. Two approximations of solutions of Hamilton-Jacobi equations. Math. Comp. 43 (1984), 1-19. Zbl 0556.65076 MR 0744921

11. Cubaud, T., \& Fermigier, M. Advancing contact lines on chemically patterned surfaces. J. Colloid Interface Sci. 269 (2004), 171-177.

12. Cubaud, T., Fermigier, M., \& Jenffer, P. Spreading of large drops on patterned surfaces. Oil \& Gas Sci. and Tech. 56 (2001), 23-31.

13. Cummings, L. J., D. Howison, S., \& King, J. R. Two-dimensional Stokes and Hele-Shaw flows with free surfaces. European J. Appl. Math. 10 (1999), 635-680. Zbl 0955.76023 MR 1757946

14. De Coninck, J., Dobrovolny, C., Miracle-Solé, S., \& Ruiz, J. Wetting of heterogeneous surfaces at the mesoscopic scale. J. Statist. Phys. 114 (2004), 575-604. Zbl 1061.82011 | MR 2035626

15. De Gennes, P.-G. Wetting: Statics and dynamics. Rev. Mod. Phys. 57 (1985), 827.

16. De Gennes, P.-G. Deposition of langmuir-blodgett layers. Coll. Polym. Sci. 264 (1986), 463-465.

17. Dietrich, S., Popescu, M. N., \& Rauscher, M. Wetting on structured substrates. J. Phys.: Cond. Matter 17 (2005), 577-593.

18. Evans, L. C., \& SprucK, J. Motion of level sets by mean curvature. I. J. Differential Geom. 33 (1991), 635-681. Zbl 0726.53029 MR 1100206

19. Freund, J. B. The atomic detail of a wetting/dewetting flow. Phys. Fluids 15 (2003), L33.

20. Friedman, A., \& HU, B. A non-stationary multi-scale oscillating free boundary for the Laplace and heat equations. J. Differential Equations 137 (1997), 119-165. Zbl 0884.35175 $\mid$ MR 1451538

21. Glasner, K. B. Spreading of droplets under the influence of intermolecular forces. Phys. Fluids $\mathbf{1 5}$ (2003), 1837-1842.

22. Glasner, K. B. A boundary integral formulation of quasi-steady fluid wetting. J. Comput. Phys. 207 (2005), 529-541. Zbl pre02183216 MR 2144627

23. Glasner, K. B. Variational models for moving contact lines and the quasi-static approximation. European J. Appl. Math. 16 (2005), 1-28.

24. Gleiche, M., Chi, L., Gedig, E., \& FuChs, H. Anisotropic contact-angle hysteresis of chemically nanostructured surfaces. CHEMPHYSCHEM 3 (2001), 187-190.

25. Gobbert, M. K., \& Ringhofer, C. A. An asymptotic analysis for a model of chemical vapor deposition on a microstructured surface. SIAM J. Appl. Math. 58 (1998), 737-752. Zbl 0926.65084 MR 1616599

26. Golestanian, R. Moving contact lines on heterogeneous substrates. Philos. Trans. R. Soc. Lond. Ser. A Math. Phys. Eng. Sci. 362 (2004), 1613-1623. MR 2084297 
27. Gorodtsov, V. A. Spreading of a film of a nonlinearly viscous fluid over a horizontal smooth surface. J. Eng. Phys. 57 (1990), 879-884. MR 1045538

28. GREEnSPAN, H. P. On the motion of a small viscous droplet that wets a surface. J. Fluid Mech. 84 (1978), 125-143. Zbl 0373.76040

29. Hadjiconstantinou, N. G. Hybrid atomistic-continuum formulations and the moving contact line problem. J. Comput. Phys. 154 (1999), 245-265. Zbl 0935.81080

30. Hocking, L. M. A moving fluid interface. Part 2. The removal of the force singularity by a slip flow. J. Fluid Mech. 79 (1977), 209-229. Zbl 0355.76023

31. Hocking, L. M., \& MiKsis, M. J. Stability of a ridge of fluid. J. Fluid Mech. 247 (1993), 157-177. Zbl 0767.76019 MR 1206141

32. Huh, C., \& Scriven, L. E. Hydrodynamic model of steady movement of a solid /liquid/fluid contact line. J. Colloid Interface Sci. 35 (1971), 85-101.

33. Hunter, J. K., Li, Z., \& Zhao, H. Reactive autophobic spreading of drops. J. Comput. Phys. 183 (2002), 335-366. Zbl 1046.76036 MR 1947775

34. Johnny, J. F., \& De Gennes, P.-G. A model for contact angle hysteresis. J. Chem. Phys. 81 (1984), 552.

35. Johnny, J. F., \& Robbins, M. Motion of a contact line on a heterogenous surface. J. Chem. Phys. 92 (1990), 3206-3212.

36. KIM, I. C. Uniqueness and existence results on the Hele-Shaw and the Stefan problems. Arch. Ration. Mech. Anal. 168 (2003), 299-328. Zbl 1044.76019 MR 1994745

37. Lipowsky, R., Brinkmann, M., Dimova, R., Franke, T., Kierfeld, J., \& Zhang, X. Droplets, bubbles and vesicles at chemically structured surfaces. J. Phys.: Cond. Matter 17 (2005), 537-558.

38. Marsh, J. A., \& CAZABAt, A. M. Dynamics of contact line depinning from a single defect. Phys. Rev. Lett. 71 (1993), 2433.

39. Moulinet, S., Guthmann, C., \& Rolley, E. Dissipation in the dynamics of a moving contact line: effect of the substrate disorder. Euro. Phys. J. B 37 (2004), 127-136.

40. Nikolayev, V. S., \& Beysens, D. A. Relaxation of nonspherical sessile drops towards equilibrium. Phys. Rev. E 65 (2002), 046135.

41. OPIK, U. Contact-angle hysteresis caused by a random distribution of weak heterogeneities on a solid surface. J. Colloid Interface Sci. 223 (2000), 143-166.

42. Oron, A., Davis, S. H., \& Bankoff, S. G. Long-scale evolution of thin liquid films. Rev. Mod. Phys. 69 (1997), 931-980.

43. Pomeau, Y. Recent progress in the moving contact line problem: a review. C.R. Acad. Sci. 330 (2002), 207-222.

44. H. Protter, M., \& Weinberger, H. F. Maximum Principles in Differential Equations. Springer, New York (1984). Corrected reprint of the 1967 original. Zbl 0549.35002 MR 0762825

45. Qian, T., WANG, X.-P., \& Sheng, P. Generalized Navier boundary condition for the moving contact line. Commun. Math. Sci. 1 (2003), 333-341. Z Zbl pre02247624 MR 1980479

46. Raphael, E., \& DE Gennes, P.-G. Dynamics of wetting with non-ideal surfaces I : The single defect problem. J. Chem. Phys. 90 (1989), 7577.

47. SCHAFFER, E., \& Wong, P. Contact line dynamics near the pinning threshold: A capillary rise and fall experiment. Phys. Rev. E 61 (2000), 5257-5277.

48. SchneEmilch, M., Quirke, N., \& Henderson, J. R. Wetting of nanopatterned surfaces: The striped surface. J. Chem. Phys. 118 (2003), 816-829.

49. Schwartz, L. W. Hysteretic effects in droplet motions on heterogenous substrates: Direct numerical simulation. Langmuir 14 (1998), 3440-3453. 
50. Schwartz, L. W., \& Eley, R. R. Simulation of droplet motion on low-energy and heterogenous surfaces. J. Colloid Interface Sci. 202 (1998), 173-188.

51. Seppecher, P. Moving contact lines in the Cahn-Hilliard theory. Internat. J. Engrg. Sci. 34 (1996), 977-992. Zbl 0899.76042

52. Voinov, O. V. Hydrodynamics of wetting. Fluid Dynamics 11 (1976), 714-721 (English translation).

53. Weidner, D. E., \& Schwartz, L. W. Contact-line motion of shear-thinning liquids. Phys. Fluids 6 (1994), 3535. Zbl 0843.76003

54. WenZel, R. N. Resistance of solid surfaces to wetting by water. Ind. Eng. Chem. 28 (1936), 988.

55. XIN, J. Front propagation in heterogeneous media. SIAM Rev. 42 (2000), 161-230. Zbl 0951.35060 MR 1778352 